\title{
DOS DEBATES Y UNA PROPUESTA PARA LA DISTINCIÓN ENTRE NEGOCIAR Y ARGUMENTAR *
}

\author{
Raúl Calvo Soler ${ }^{1}$ \\ Universidad de Girona
}

RESUMEN. El artículo desarrolla un concepto de negociación a partir de una específica combinación de modos, entendidos como formas de finalización de un conflicto, e instrumentos, concebidos como los recursos que pueden usar los actores para resolver un conflicto. También se considera la relación que media entre el concepto de negociación y los de argumentación y diálogo. Además, se analizan dos debates posibles en torno al concepto de negociación propuesto; el tema de la relación entre la negociación y los diferentes tipos de argumentación y el análisis de los supuestos de no negociabilidad.

Palabras clave: negociación, conflicto, argumentación, negociabilidad.

ABSTRACT. The article develops a concept of negotiation from a specific combination of modes, understood as forms of ending of a conflict, and instruments, conceived as the resources that the actors can use to solve a conflict. The relationship between the concept of negotiation and those of argumentation and dialog is also considered. In addition, the article analyzes two possible debates around the concept of negotiation proposed, as well as the relationship between negotiation and the different types of argumentation, and also the cases of non-negotiability.

Keywords: negotiation, conflict, argumentación, negotiability.

* Fecha de recepción: 7 de enero de 2008. Fecha de aceptación: 31 de enero de 2008.

1 Este trabajo ha sido posible gracias a la ayuda del proyecto de investigación del Ministerio de Educación y Ciencia SEJ2004-07136-02/JURI. 


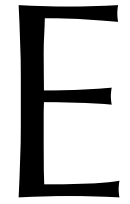

1 objetivo de este trabajo es presentar una propuesta conceptual de la negociación que permita distinguirla, por un lado, de otros procesos de resolución de conflictos como el diálogo o la deliberación y, por el otro lado, de uno de los instrumentos que pueden ser usados por los actores en estos procesos: la argumentación.

Esta propuesta conceptual apunta a su vez a dos objetivos ulteriores que sólo serán tangencialmente considerados en este trabajo: 1) establecer las implicaciones prácticas que se siguen de la utilización de un método u otro, esto es, definir a qué nos estamos comprometiendo al postular el desarrollo de un proceso de negociación o un proceso de diálogo en diferentes ámbitos, y 2) presentar un análisis comparativo respecto de las virtudes y defectos que ostentan cada uno de ellos en referencia a diferentes finalidades.

Sin embargo, antes de presentar esta propuesta creo conveniente realizar tres consideraciones con el objeto de aclarar el contexto en el que voy a desarrollar el análisis.

\section{ARGUMENTACIÓN Y NEGOCIACIÓN}

No es extraño que en los ámbitos en los que ha surgido la confrontación entre estos dos métodos suela contraponerse a la negociación la noción de «argumentación». En mi opinión, y por razones que desarrollaré más extensamente en este trabajo, esta contraposición puede conducir a ciertos equívocos en relación con dos categorías distintas. Por un lado, está el tema de los procesos o métodos que, siguiendo a VON WRIGHT, son hechos que se caracterizan por su continuidad ${ }^{2}$ en el tiempo. A esta categoría pertenecen, en mi opinión, las nociones de negociación y diálogo. Pero, por el otro lado, están aquellos elementos que en el contexto de un proceso o método determinado son usados por los actores. Éste es el caso, por ejemplo, de la promesa, la amenaza, el compromiso, la advertencia y los argumentos.

Cuando se establece la contraposición entre argumentación y negociación surgen dos cuestiones problemáticas: en primer lugar, está la cuestión de en qué medida la noción de argumentación recepta adecuadamente el aspecto de continuidad de los procesos. Y, en segundo lugar, aparece la duda acerca de si se está distinguiendo claramente entre el tema del proceso y el de los elementos o instrumentos usados en él. Para evitar estas posibles confusiones plantearé mi análisis en referencia a la contraposición entre negociación y diálogo y dejaré el término de argumentación para referirme al plano de los elementos usados en un proceso.

\section{NEGOCIACIÓN, DIÁLOGO Y RESOLUCIÓN DE CONFLICTOS}

En mi opinión, cuando se pretende establecer un análisis comparativo entre estos dos métodos es importante tener en cuenta la diferencia de alcance o amplitud de cada uno de ellos. Creo que sea cual sea la definición que se proponga del proceso negocial ésta está

2 G. H. von Wright, 1971: Norma y acción, Madrid: Tecnos, pp. 44 y 45. 
ineludiblemente vinculada a la preexistencia de un conflicto, o dicho de otra manera, me resulta difícil de comprender la noción de negociación al margen de su consideración en términos de un método de solución de conflictos ${ }^{3}$. Por el contrario, creo que la caracterización de los procesos de diálogo y deliberación no está ineludiblemente vinculada al tema de la búsqueda de una solución del conflicto sino que puede ir más allá. Por ejemplo, para decirlo con la terminología de la teoría de juegos, y aunque seguidamente volveré con mayor detalle sobre las nociones de conflicto y de solución, creo que tiene perfecto sentido sostener la existencia de un proceso de diálogo en el contexto de un juego de pura coordinación ${ }^{4}$; podemos dialogar para tomar decisiones en escenarios no conflictivos.

Si se acepta esto, resulta importante que al caracterizar la noción de proceso de diálogo para un futuro desarrollo comparativo lo hagamos a partir del ámbito del proceso más específico, esto es, tomando en consideración las propiedades del diálogo relacionadas con su caracterización como un método de solución de conflictos. En caso contrario corremos el riesgo de asignar a éste proceso ciertas virtudes frente a las cuales el proceso de negociación no puede decir nada.

\section{LA CARGA EMOTIVA NEGATIVA DE LA NEGOCIACIÓN}

El debate entre el proceso de negociación y el de diálogo es, por momentos, un debate dispar. Esta disparidad viene dada fundamentalmente por la diferente carga emotiva con la que cuentan cada uno de estos términos; la negociación tiene una carga emotiva negativa, mientras que el diálogo cuenta con una carga emotiva positiva. Ahora bien, en mi opinión, el problema de la disparidad se plantea en tanto que esta carga emotiva, especialmente la referida a la negociación, pareciera ser suficiente como para que muchos autores, por no decir la mayoría, que han discutido sobre estas temáticas despachen la cuestión de la definición de la negociación y de los defectos de ésta con un mínimo de discusión. Por ejemplo, es curioso notar cómo en muchas de las publicaciones que presuponen la distinción conceptual entre negociación y diálogo hay una patente falta de referencias respecto de la importante literatura sobre la negociación como método de resolución de conflictos 5 .

3 Un ejemplo claro de que muchas veces el debate gira en torno a la cuestión de las decisiones colectivas y no estrictamente sobre las decisiones colectivas conflictivas puede verse en J. L. MARTí, 2006: La república deliberativa. Una teoría de la democracia, Madrid-Barcelona: Marcial Pons. El autor insiste con claridad, a partir del trabajo de ELSTER, en la reconstrucción del principio de negociación como un principio democrático de toma de decisiones (pp. 41 y ss.). La cuestión clave, y sobre la que insistiré en este trabajo, es si todas las virtudes atribuidas a la argumentación pueden seguir siendo sustentadas cuando ésta está referida en exclusividad al ámbito de las decisiones colectivas conflictivas.

${ }^{4}$ La teoría de juegos distingue tres clases de juegos: los juegos de puro conflicto, los juegos de pura coordinación y los juegos mixtos. Los juegos de puro conflicto, que incluirían como una subclase a los conocidos juegos de suma cero, se caracterizan porque los intereses de los jugadores son opuestos. Esta idea responde al enunciado «el aumento de la utilidad de un movimiento por parte de un jugador depende de la pérdida de utilidad por parte del otro u otros jugadores» o en otros términos «lo que uno gana lo pierde el otro y lo que el otro gana lo pierde uno». Por el contrario, los juegos de pura coordinación se caracterizan porque los intereses de los jugadores son comunes. Esto puede representarse mediante la idea de «siempre que yo gano ganas tú y siempre que yo pierdo pierdes tú». Finalmente, un juego es mixto si combina perfiles de juegos de puro conflicto y perfiles de pura coordinación.

5 Un ejemplo paradigmático de este tipo de situaciones es la compilación de J. ELSTER, 2001: La democracia deliberativa, Barcelona: Gedisa. En diferentes artículos de esta compilación se hace referencia a la negocia- 
Si vamos a caracterizar la negociación, me parece que será importante comprender cómo definen la negociación aquellos que la tienen como objeto de estudio, aunque sea como un presupuesto para posteriormente mostrar los problemas que plantea esa definición y proponer una alternativa. En caso contrario, creo que se corre el riesgo, como a veces parece ocurrir en este debate, de presentar una definición de negociación que ninguno de los investigadores que trabajan en este campo aceptaría y que suele ser una caricaturización que muestra la peor presentación posible del proceso negocial.

\section{ALGUNOS ÁMBITOS DEL DEBATE}

La confrontación entre los procesos negociales y los procesos de diálogo ha surgido en una pluralidad de ámbitos temáticos de entre los cuales quizás quepa resaltar los tres siguientes:

1. El ámbito de los conflictos internacionales. En este contexto se suele plantear la discusión en referencia a cuál es el mejor método para avanzar en la consecución de acuerdos que permitan a las partes superar la contienda.

2. Procesos de paz con movimientos terroristas. También en este espacio de discusión ha surgido la cuestión entre los dos procesos. En este segundo caso la clave del debate normalmente gira en torno a la legitimidad que cada uno de estos métodos otorga a un proceso de paz teniendo en cuenta los límites que impone la condición del Estado de Derecho.

3. La democracia deliberativa. En este último ámbito se suele encarar el problema de los mecanismos de toma de decisiones colectivas y la cuestión acerca de cuál de éstos potencia las características propias de los sistemas democráticos.

Hay una diferencia sustancial, que ya he mencionado con anterioridad, entre los dos primeros ámbitos y el último: el rol del conflicto. En los dos primeros se parte con absoluta claridad de la idea de conflicto y se consideran los dos procesos en términos de métodos de solución de estos conflictos. Sin embargo, en el tercero de estos ámbitos no siempre la distinción resulta clara. Como he sostenido anteriormente, en mi opinión no todo problema de decisión colectiva, que es normalmente el ámbito en el que desarrollar el debate de la democracia deliberativa, implica la existencia de un conflicto. Para evitar posibles confusiones comenzaré desarrollando las nociones de conflictos y de solución de conflictos en función de las cuales presentaré la distinción conceptual entre el proceso de negociación y el de diálogo.

\section{CONFLICTO Y SOLUCIÓN DE CONFLICTOS}

La caracterización de lo que ha de ser entendido por «conflicto» no es una cuestión sencilla. Diferentes teorías han puesto el acento en distintos rasgos para delimitar una

ción en términos negativos. A modo de ejemplo se relaciona la negociación con la manipulación (p. 18), la tergiversación de las preferencias (p. 19) o el contubernio (p. 20). Sin embargo, cabe resaltar que no hay en todo el libro una cita de algún texto vinculado con el estudio de los procesos negociales ni hay un mayor intento de los participantes en sustentar o defender estas afirmaciones. 
definición de este fenómeno tan complejo. No es mi intención realizar aquí un análisis exhaustivo de las distintas categorías que han sido presentadas para dar cuenta del concepto de conflicto ${ }^{6}$. Centraré mi análisis en los aspectos que considero más relevantes.

El punto de partida que utilizaré para definir «conflicto» son las relaciones sociales ${ }^{7}$, esto es, un comportamiento recíproco de dos o más individuos que orientan, comprenden y resuelven sus conductas teniendo en cuenta las de los demás, con lo que dan sentido a sus actos ${ }^{8}$. De esta idea me parece importante señalar dos aspectos:

1. Comportamiento recíproco. La idea de comportamiento recíproco hace referencia a la vinculación de las conductas de los sujetos. De esta manera, lo que el otro hace supone una afectación a lo que yo haré. Hablamos en este sentido de «comportamiento recíproco» en términos de interacción entre dos o más actores. Por supuesto, la cuestión que queda por dilucidar es el criterio definitorio de una situación de interacción. Volveré seguidamente sobre este aspecto.

2. Tener en cuenta la conducta de los otros. La idea de tener en cuenta parece apuntar, fundamentalmente, a la cuestión de la intencionalidad de las conductas. Puede ser que un determinado comportamiento afecte, de alguna manera, a un determinado actor. Pero, si esa afectación no fue realizada de forma intencional, no habrá interacción social. Por ejemplo, supóngase que choco mi auto con otro. Hasta aquí no hay interacción social y, por ende, no hay conflicto en tanto que la afectación entre las conductas se ha producido de forma fortuita. Ahora bien, cuando yo veo que el otro conductor sale del auto realizando gestos de desaprobación, puedo generar una interacción social si decido cómo actuaré a partir de cómo actuó el otro. Si por el contrario, salgo del coche sin observar al otro y mi acción no toma en cuenta la conducta (o potencial conducta) del otro conductor, no habrá interacción social entre nosotros?

De las diversas formas de agrupar las relaciones sociales, el criterio de la «incompatibilidad de objetivos» como definitorio de la interacción ha resultado especialmente prolífico. A partir de este criterio es posible diferenciar dos tipos de relaciones sociales: relaciones sociales donde los actores sustentan la consecución de objetivos que son compatibles y aquéllas en las que los objetivos son incompatibles:

1. Relaciones sociales con objetivos compatibles. Dos objetivos son compatibles cuando la satisfacción de uno de ellos por parte de un actor no afecta a la satisfacción del objetivo de otro actor. En adelante denominaré a este tipo como relación social cooperativa puesto que en estos casos no queda excluida la relación de cooperación entre actores.

2. Relaciones sociales con objetivos incompatibles. Dos objetivos son incompatibles cuando la satisfacción de uno de los objetivos de un actor supone la no satisfacción del objetivo del otro actor. En adelante denominaré a este tipo como relación social conflictiva.

${ }^{6}$ Puede verse un análisis pormenorizado de las diferentes teorías en J. A. SCHELLENBERG, 1996: Conflict Resolution, New York: State University of New York Press.

7 Por todo véase J. Freund, 1995: Sociología de los conflictos, Madrid: Ministerio de Defensa. Secretaría General Técnica.

8 M. Weber, 1992: Economía y Sociedad, México: Fondo de Cultura Económica. Trad. de José Media Echavarria.

9 Sobre el tema de la intencionalidad en la definición de las relaciones sociales según la propuesta de WEBER véase la propuesta del autor así como los ejemplos utilizados. Por todo, M. WEBER, 1992: Economía y Sociedad, op. cit., pp. 21 y ss. 
En lo que sigue consideraré como conflicto una relación social en la que dos o más sujetos sustentan objetivos incompatibles o percibidos como incompatibles.

Una vez desarrollada la noción de «conflicto» que usaré en este trabajo es necesario analizar qué se entiende por «solucionar un conflicto».

Es un lugar común en el discurso de los conflictos que puede intervenirse en tres planos distintos: intervenir para prevenir, para gestionar o para solucionar conflictos. La distinción entre el primer supuesto y los otros dos es clara; en el primero el conflicto aún no ha aparecido y la intervención puede tener dos objetivos distintos: intervenir para corregir aquellos elementos que causarían el surgimiento del conflicto (evitar el conflicto) o intervenir para encauzar dichos elementos de forma tal que el conflicto surja de una manera controlada (conducir el conflicto). En el caso de la distinción entre gestión y solución la distinción es algo más controvertida. En principio, la intervención para gestionar supone que el conflicto ya ha surgido (existe la percepción de al menos uno de los actores de estar en un conflicto) pero incluye ciertos elementos que obstaculizan cualquier posible avance hacia su solución. Por ejemplo, uno de los actores no reconoce al otro como actor o ambos consideran que lo que está en juego es una cuestión de principios o valores que no puede ser resuelta. En este sentido, la gestión supone una intervención para conducir ciertos aspectos del conflicto hacia un estadio en el que sea posible iniciar la intervención en términos de resolución ${ }^{10}$. Finalmente, la intervención puede estar centrada en la solución del conflicto, esto es, en la búsqueda e identificación de aspectos que permitan la superación de la incompatibilidad y la configuración de un nuevo tipo de relación social entre las partes.

Una de las cuestiones que han animado el debate de las teorías de conflictos ha sido el referido a cuándo cabe considerar que un conflicto ha concluido. La historia de las relaciones, tanto internacionales como personales, está llena de casos en los que un conflicto surgido en un tiempo presente se encuentra directamente relacionado con algún conflicto de tiempos pasados. Esta vinculación se genera con independencia de la amplitud del lapso que media entre estos dos momentos. Por ejemplo, es un dato conocido que la firma del tratado de rendición de Francia durante la Segunda Guerra Mundial se realizó en el mismo vagón de tren, que fue expresamente transportado hasta París, en la que los franceses hicieron firmar a los alemanes la rendición en la Primera Guerra Mundial. No tratándose de una cuestión meramente estética, hasta qué punto se puede afirmar que en casos como éste estamos ante el mismo conflicto es una cuestión que no trataré en este trabajo. En cambio, sí me parece importante establecer algunas concreciones sobre el concepto de solución de un conflicto.

La idea de solución de un conflicto, si se acepta la definición de conflicto anteriormente presentada, está vinculada con la consecución de los objetivos por parte de alguno o de todos los actores implicados en él. En este sentido es importante señalar tres propiedades de esta noción de conclusión de un conflicto:

a) Esta noción no implica la consecución conjunta de los objetivos de todos los actores del conflicto; un conflicto puede concluir porque uno de los actores consiga su

${ }^{10}$ Puede verse un desarrollo específico sobre el tema de la gestión de conflictos en referencia al control de la escalada (proceso de aumento de la intensidad en los recursos que utilizan los actores del conflicto) en D. G. PruitT y S. H. Kim, 2004: Social Conflict: Escalation, Stalemate and Settlement, Boston: Mac Graw Hill. 
objetivo en detrimento del otro actor al carecer este último de recursos para mantener la defensa de su objetivo.

b) La noción de solución no requiere el mantenimiento de los objetivos por parte de los actores y acepta la posibilidad del cambio de éstos; un conflicto puede concluir por la modificación de los objetivos de uno o varios actores.

c) Un conflicto puede solucionarse por la eliminación de alguno de los elementos constitutivos (por ejemplo, la eliminación de uno de los actores del conflicto o la desaparición de aquello que es objeto del conflicto) en cuyo caso me referiré a esta situación como proceso de disolución del conflicto. O mediante la consecución de un acuerdo entre los actores sin que se haya producido la eliminación de alguno de estos elementos (por ejemplo, por la consecución de un acuerdo entre los actores). Me referiré a este último supuesto como caso de resolución del conflicto.

A partir de la combinación de estas tres características es posible establecer una clasificación de lo que se ha dado en llamar modos de un conflicto.

\section{MODOS DEL CONFLICTO}

Cuando se habla de los modos se está haciendo referencia al tipo de resultado o a la forma como concluye el conflicto. La clasificación más tradicional de los modos en que puede concluir un conflicto se limita a cuatro supuestos: 1) imposición; 2) retirada o desistimiento; 3) conversión o allanamiento, y 4) compromiso ${ }^{11}$.

1. Imposición. Un conflicto termina por imposición cuando uno de los actores logra la consecución total de sus objetivos frente al otro actor, el cual ve frustrada la obtención de los suyos. La imposición supone entonces la prevalencia de los objetivos de una de las partes frente a los de la otra sin que el otro deje de considerarlos como objetivos (no hay modificación).

2. Retirada o desistimiento. Un conflicto termina por retirada cuando uno de los actores logra la consecución total de su objetivo frente al otro actor, que abandona la confrontación y la defensa de sus reclamaciones. En este segundo caso, y a diferencia de la imposición, la prevalencia de los objetivos de uno de los actores se produce porque el otro renuncia a la defensa de su objetivo, aunque no hay una modificación de éstos.

3. Conversión o allanamiento. Un conflicto termina por conversión cuando uno de los actores asume como suyos los objetivos defendidos por el otro actor. Este tercer caso se diferencia de los dos anteriores en el hecho de que desaparece uno de los objetivos incompatibles: el del sujeto que se allana o convierte. Especialmente, la diferencia con el caso del desistimiento es que la modificación de la situación no supone que el actor que se allanó siga defendiendo su objetivo.

4. Compromiso. Un conflicto termina por compromiso a partir del intercambio de concesiones entre los actores. La concesión es posible a partir de un conjunto de in-

${ }^{11}$ La caracterización que aquí presento de esta clasificación difiere de la que tradicionalmente se ha venido utilizando. El problema que plantea en mi opinión la propuesta tradicional es que construye los modos de forma no excluyente. Al introducir en la definición la no renuncia de la otra parte se salva este inconveniente. Para una visión de la forma clásica de presentar estas categorías véase R. ENTELMAN, 2001: Teoría de conflictos. Hacia un nuevo paradigma, Barcelona: Gedisa. 
dividualizaciones diferentes de un mismo objetivo genérico. Por ejemplo, si mi objetivo es comprar una casa por no más de 200.000 euros, la concesión se produce cuando frente a una primera oferta de 150.000 euros estoy dispuesto a pagar 160.000 .

Ahora bien, una cuestión sobre la que han insistido los autores que trabajan sobre el conflicto es la de si cabe establecer algún tipo de relación entre los modos de finalización de un conflicto y los métodos de resolución. En este trabajo defenderé que efectivamente existe esta relación y, además, que ésta es de carácter conceptual, esto es, que un elemento definitorio de los diferentes métodos de solución de un conflicto es el modo o modos a los que conducen la utilización de cada uno de ellos.

Pero, para poder completar el conjunto de elementos que conforman la definición de un método de solución de conflictos es necesario introducir un segundo elemento: los instrumentos.

\section{INSTRUMENTOS DE SOLUCIÓN DE CONFLICTOS}

Entiendo por instrumentos de la solución de un conflicto al conjunto de recursos que las partes pueden utilizar para conseguir sus objetivos. Los ejemplos de instrumentos en la solución de un conflicto son múltiples y variados y van desde el armamento, pasando por las promesas, los compromisos, las advertencias y las amenazas, hasta llegar a los argumentos.

Sin embargo, dentro del amplio abanico de posibles instrumentos hay dos que, en lo que aquí interesa, conforman dos referencias clásicas cuando nos referimos al tema del diálogo y de la negociación: las amenazas y la argumentación. Veamos someramente cada uno de ellos.

La amenaza es un instrumento de tipo coactivo que pretende condicionar la decisión del interlocutor a partir de un cálculo fundamentado exclusivamente en el análisis de costos y beneficios. La idea del sujeto que emite la amenaza es la siguiente: la propuesta de solución del conflicto respaldada en este elemento coactivo tiene sentido para el otro actor en tanto que el costo que para él significará que se cumpla la amenaza es mayor que el beneficio de rechazar la oferta de solución que se le realiza.

Lo relevante para entender el funcionamiento de este instrumento es que la amenaza actúa con independencia de las razones que puedan darse a favor de la aceptación de la propuesta. En este sentido, es irrelevante si el sujeto que amenaza tiene buenos argumentos para respaldar la idoneidad de su propuesta; se puede emitir una amenaza aunque no haya ninguna razón que justifique o explique por qué es interesante la propuesta como una forma de finalizar con el conflicto. Dicho en otros términos, la fuerza de la propuesta se agota en los costos que traería aparejados el cumplimiento de la amenaza.

Por su parte, los argumentos, en tanto que un instrumento para la resolución de conflictos, pretenden producir una reflexión en el interlocutor; se trata de explicar o justificar la propuesta que se hace. Las razones funcionan, en el contexto de la solución de conflictos, como fundamentos acerca de la idoneidad de la propuesta como un marco de superación de la controversia. 
Así planteada, la distinción entre argumentos y amenazas radica en que mientras que las primeras están relacionadas (a favor o en contra) con el contenido específico de la solución del conflicto, las segundas, con ser relevantes para entender la dinámica de solución del conflicto, carecen de referencia en lo que hace propiamente a su contenido ${ }^{12}$.

Aunque volveré posteriormente sobre algunas cuestiones vinculadas con el tema de la relación entre argumentación, amenazas y negociación creo que es posible con estos elementos presentar dónde radica la diferencia entre los procesos de negociación y de diálogo.

\section{LA DEFINICIÓN DEL MÉTODO}

En lo que sigue voy a considerar la definición del método de la negociación en términos de una específica combinación de modos e instrumentos.

En líneas generales podría decirse que las característica que mayoritariamente los investigadores de este ámbito le atribuimos a la negociación son tres: $a$ ) es un método cuyo objetivo es la expectativa de encontrar una solución para un conflicto; $b$ ) en el que tiene sentido el uso de los instrumentos de corte coactivo (la amenaza, el compromiso, la advertencia) que operan más allá del contenido de la solución, y c) en donde el valor de la solución pasa únicamente por la voluntad de los actores ${ }^{13}$. Veamos cada una de estas propiedades.

\subsection{La expectativa de solución del conflicto}

En primer lugar, en una negociación las partes implicadas tienen una expectativa, más o menos fundada, de que el proceso les conducirá a un escenario en el que podrán superar el conflicto. Por supuesto, esta expectativa puede fallar y el proceso concluir sin que se haya modificado la situación conflictiva. En estos casos diremos que la negociación ha fracasado.

Esta primera característica se utiliza para distinguir lo que sería un proceso de negociación propiamente dicho de los casos en los que los actores acuden al proceso simplemente por razones estratégicas; para saber qué información tiene el otro, para dilatar en el tiempo el proceso judicial, para disuadir al otro de que inicie un juicio, etcétera.

12 Ahora bien, hay otro instrumentos que en él ámbito de la solución de conflictos parecen constituir una categoría intermedia entre la amenaza y la argumentación: las advertencias. Por ejemplo, supongamos que un acreedor sostiene algo como lo siguiente: «si no me pagas ahora el 70 por 100 de la deuda, no podré seguir produciendo en la cantidad que lo he hecho hasta ahora porque necesito ese dinero y tendré que dejar de suministrarte productos». ¿Es esto una amenaza o un argumento? No me ocuparé aquí de esta cuestión ya que a los efectos de este desarrollo no es relevante.

13 Hay una cuarta propiedad que suele introducirse a la hora de definir la negociación: no hay ningún tercero que intervenga en el proceso, esto es, alguien que no es un actor o representante de un actor del conflicto. En todo caso ésta es común al diálogo así que no será aquí considerada. 


\subsection{El uso de las amenazas}

Prácticamente la totalidad de modelos de negociación y de autores que discuten en torno a este método aceptan como un elemento indefectiblemente vinculado al proceso negocial el uso de instrumentos coactivos, como por ejemplo la amenaza. Ahora bien, me parece que no hay que confundir el reconocimiento del rol que juegan las amenazas en el proceso de negociación con: 1) la afirmación según la cual la ausencia de amenazas indefectiblemente niega la condición de negociación al proceso, y 2) la afirmación según la cual no es posible el uso de argumentos en el contexto negocial.

Lo que aquí sostengo es que la identificación del proceso negocial depende de una actitud interna de los actores conforme a la cual tiene sentido el uso de estas tácticas coactivas en el proceso. Si alguien está interactuando para solucionar un conflicto y no acepta la posibilidad de usar las amenazas, entonces no está negociando. Pero esto no dice nada acerca de la posibilidad de que dos actores negocien, lleguen a un acuerdo y finalicen el conflicto sin que de hecho hayan efectivizado las amenazas que estaban dispuestos a usar.

Un ejemplo característico de procesos negociales en los que no hay efectivización de las amenazas son las negociaciones de una tirada. En este tipo de negociaciones lo que suele ocurrir es que la oferta de uno de los negociadores supera con creces las expectativas iniciales del otro negociador. En este punto, una fuerte animadversión al riesgo, por ejemplo, puede conducir a la aceptación de la oferta sin que concurran amenaza o argumentación alguna.

Tampoco el reconocimiento del rol de la amenaza en los procesos negociales dice nada acerca de la ausencia del uso de los argumentos en la negociación. El rol de la argumentación en los contextos negociales ha adquirido una gran trascendencia en lo referente al ámbito del regateo. En este sentido, resulta ilustrativa la distinción entre ofertas y propuestas. Se entiende por oferta a una potencial solución del conflicto expresada por uno de los negociadores. Por ejemplo, pagar en dos meses el 100 por 100 de la deuda, establecer un régimen de visitas para los fines de semana o la entrega del armamento del que dispone un país. La aceptación de una oferta por parte del otro negociador implica automáticamente la constitución de un acuerdo que supone la resolución total o parcial del conflicto. Por su parte, se entiende por propuesta a una oferta que incluye la totalidad de premisas que la explican o justifican. Por ejemplo, pagar en dos meses el 100 por 100 de la deuda porque el ciclo de mi negocio requiere de ese tiempo para poder cancelar la deuda.

Los casos en los que concurre la argumentación en una negociación pueden ser de dos tipos: en primer lugar, está el caso en que uno de los dos negociadores presenta una propuesta en la mesa de negociación. En segundo lugar, está el caso en que habiendo realizado el negociador una oferta se le reclama la explicitación de las premisas explicativas y/o justificativas de dicha oferta, es decir, la conversión de la oferta en una propuesta.

Si se acepta esto, entonces la relación entre instrumento y método puede ser resumida de la siguiente manera:

1. Es condición necesaria para la negociación la actitud interna respecto al sentido que tiene el uso de la amenaza en el proceso de resolución. 
2. No es condición necesaria para referirnos a la negociación la ausencia del uso de argumentos.

Por supuesto, un caso extremo de procesos negociales son aquellos en los que la amenaza no se efectiviza ni hay argumentos entre las partes. El ejemplo paradigmático de este tipo de situaciones son las denominadas negociaciones tácitas, esto es, «...aquella en la que la comunicación es incompleta o imposible...» ${ }^{14}$.

Ahora bien, llegado este punto surgen dos cuestiones que considero relevantes en la medida en que pueden condicionar lo hasta aquí desarrollado: el tema de los tipos de argumentación y la cuestión de lo no negociable.

La primera de estas cuestiones creo que puede ser planteada en los siguientes términos: si bien es cierto que en los procesos negociales puede concurrir la argumentación, es necesario acotar que ésta tiene características diferenciales respecto de la argumentación que se da en el plano de otros métodos, como por ejemplo el diálogo. La diferencia sustancial entre ambos tipos de argumentación radica en la actitud de aquel que ofrece los argumentos. En la negociación la actitud interna de quien ofrece razones es siempre de corte egoísta (no imparcial), sus argumentos aunque externamente puedan parecer imparciales, responden a un único parámetro: la búsqueda de la satisfacción de sus propios intereses ${ }^{15}$. Por el contrario, cuando la argumentación se da en otros ámbitos el conjunto de razones ofrecidas responde, en la actitud interna, a un esquema de imparcialidad.

El problema fundamental que plantea una objeción de estas características es el concepto de «imparcialidad» que se utiliza ${ }^{16}$. En mi opinión en este contexto caben tres posibles interpretaciones de la noción de imparcialidad.

La primera posibilidad es sostener que la ausencia de imparcialidad significa que cada actor construye sus ofertas para la resolución del conflicto sin tener en cuenta los intereses de la otra parte. Si es ésta la noción de imparcialidad que se está manteniendo, me parece que la crítica no se sostiene. Muchos modelos de negociación ${ }^{17}$ incorporan un conjunto de metodologías (empatía, cambio de roles, búsqueda de criterios externos, escucha activa) cuyo último objetivo es la presentación de propuestas que recepten los intereses de todas las partes participantes en el conflicto. En este sentido, un detentador de los modelos de negociación basados en estos requisitos procederá, en el aspecto externo, a construir propuestas sustentadas en razones que recepten los intereses de ambas partes, y, en el aspecto interno, lo hará en la medida en que concibe al proceso negocial como una búsqueda de la mejor solución para ambas partes. Por

14 Para un desarrollo pormenorizado de este tipo de negociaciones véase Th. SCHELLING, 1971: La estrategia del conflicto, Madrid: Tecnos, pp. 71 y ss.

15 Creo que una excelente presentación de este tipo de objeciones es la presentada por Cristina REDONDO en un artículo no publicado titulado «Sobre la fuerza civilizadora de la argumentación estratégica». En este artículo puede leerse: «Desde este punto de vista, cabe distinguir entre quien ofrece razones imparciales con la actitud interna típica de quien negocia (buscando satisfacer intereses egoístas) y quien lo hace con una actitud que mira a la comprensión y que está dispuesto a aceptar la conclusión avalada por las mejores razones» (p. 3)

16 En el citado artículo de Cristina REDONDO se reconoce la problemática que plantea la definición del requisito de imparcialidad.

17 Existe una importantísima bibliografía en este sentido pero a modo de ejemplo puede citarse URY, BRETT y GolberG, 1988: Getting Disputes Resolved, San Francisco: Jossey-Bass. 
supuesto, es posible que algunos negociadores pretendan sacar partido participando de estos procesos mediante un planteamiento puramente hipócrita, sin la convicción que requiere el aspecto interno. Pero, esto es contingente y en todo caso nada dice acerca de si la negociación puede receptar o no este tipo de argumentación ${ }^{18}$.

Una segunda forma de reconstruir la imparcialidad sería la siguiente: que las razones sean imparciales quiere decir que los actores (internamente) sustentan argumentos motivados por la búsqueda de una solución que es independiente a los intereses de las partes que conforman el conflicto, esto es, hay intereses fuera del conflicto que de ser la argumentación imparcial deberían incorporarse al discurso de la solución. Si es ésta la interpretación de la imparcialidad creo que cabría aceptar que la negociación presupone y está limitada por el juego de los intereses que efectivamente concurren en el conflicto. Para decirlo en el caso extremo no tiene sentido postular conceptualmente como proceso negocial un ámbito en el que las partes buscan soluciones a un conflicto con independencia de los intereses que conforman.

Sin embargo, creo que el reconocimiento de este punto importa en realidad una consideración menor de lo que a primera vista pudiera parecer en lo referente al rol y trascendencia del método negocial. Esto es así porque creo que hay un punto en el que ambas nociones pueden colapsar.

Partamos de la aceptación de que en los procesos de negociación es posible construir propuestas de solución sustentadas en argumentos que consideran relevante, tanto externa como internamente, que ésta recepte todos los intereses implicados en el proceso. Pues bien, si esto es así, la posibilidad de que la argumentación sea imparcial en el segundo de los sentidos dependerá simplemente de que todos los intereses empíricamente dados estén receptados en el conflicto. En este sentido, la argumentación en la negociación vulnerará el reclamo de imparcialidad sólo en aquellos casos en que existan intereses que no estén adecuadamente incorporados en el escenario conflictivo. Por lo tanto, en el proceso negocial habrá casos de argumentación imparcial y casos de argumentación no imparcial.

Lo que en todo caso sí me parece aceptable de esta disquisición es que en aquellos supuestos en los que ciertos intereses queden fuera del halo del conflicto, la negociación, en tanto que un método de solución, no exigirá su incorporación a los efectos de evaluar la adecuada o pertinente finalización del conflicto.

Hay finalmente una tercera posibilidad de establecer la imparcialidad; un argumento es imparcial cuando es independiente de cualquier interés sea que éste concurra o no en el conflicto. Si es esto lo que se está sosteniendo me parece que también cabría aceptar la matización; en el proceso negocial la idea de solución y de valoración de la solución está condicionada por los intereses de los actores (de las partes o de terceros).

Pero, una propuesta de estas características creo que exige, al menos, la respuesta a dos preguntas por parte de aquellos que la sostienen; si se mantiene la caracterización de la negociación como un método de solución de conflictos, sería necesario

18 Ya he defendido en otro lugar que este tipo de consideraciones sólo puede ser defendido a partir de una caricaturización de los procesos de negociación. Por todo véase «Entre ángeles y demonios anda el juego», en www.latrama.com. 
identificar, por un lado, ¿qué noción de conflicto sostiene una propuesta como la anteriormente expresada? Y, por el otro lado, ¿a qué le estamos llamando solución del conflicto? Sinceramente, no tengo claro que una respuesta a estas cuestiones no vaya en detrimento de la caracterización de la negociación como un método de solución de conflictos.

La segunda de las cuestiones que condiciona el alcance de la caracterización del proceso negocial podría ser planteada en los siguientes términos: aunque se aceptase que en el proceso de negociación cabe el uso de argumentos existen una cantidad de elementos que pueden ser relevantes para la solución de un conflicto que no son susceptibles de una solución negociada. En este sentido, habría un ámbito para la argumentación que necesariamente excluiría a la negociación entre las partes; dicho de otra manera, cuando las partes tienen un conflicto en estos ámbitos no pueden negociar.

Una forma tradicional de establecer la cuestión de lo no negociable ha sido tomar en consideración ciertas propiedades de las conclusiones del argumento en cuestión. Por ejemplo, un autor como ELSTER sostiene que no se puede negociar sobre cuestiones de hecho «...de la misma manera que uno no puede negociar sobre el clima...» ${ }^{19}$.

Ahora bien, me parece que esta intuitiva afirmación de ELSTER requiere un análisis más detallado. ¿Qué quiere decir que no se puede negociar sobre el clima? En mi opinión, caben cuatro respuestas posibles a esta cuestión:

1. No se puede negociar sobre el clima porque no es posible un conflicto sobre el clima, en el sentido anteriormente expresado; la producción de la lluvia, del sol o de la niebla no depende de nuestras acciones con lo que difícilmente puede darse una situación de interacción entre los actores. Pero lo que sí se puede hacer es argumentar en referencia a la producción de estos fenómenos atmosféricos.

Es importante señalar que, al menos en lo referente al clima, esta imposibilidad es fundamentalmente técnica (ni lógica, ni física). En el mismo momento en que esto sea posible podremos considerar al clima como objeto de una negociación, si es que, en algún sentido no estamos ya en ese momento en el que el hombre puede, si no controlar, al menos condicionar con sus acciones el clima.

Más allá de la cuestión del clima me parece que lo que sí cabe reconocer es que no es posible una negociación en los casos en los que la satisfacción de los objetivos de uno de los actores no depende de las acciones del otro actor. Por ejemplo, yo no puedo negociar la venta de la empresa Coca-Cola si esto no depende de mis acciones. Por supuesto, el término «dependencia» puede plantear casos difíciles en los que tengamos problemas para saber si un determinado estado de cosas del mundo depende o no de mis acciones. Pero no me ocuparé aquí de esta cuestión.

2. Una segunda forma de entender la idea de «no negociabilidad del clima» es pensar que lo que no es susceptible de negociación es el valor de verdad del enunciado referido al clima, es decir, no tiene sentido afirmar que vamos a negociar «si el enun-

19 J. ElSTER, 1995: «Strategic Uses of Argument», en ARROw, MNOOKIn, Ross, TVERSKY y WiLSON (eds.), Barriers to Conflict Resolution, New York: Norton, p. 238 (la traducción es mía). 
ciado "está lloviendo" es verdadero o falso», aunque sí tendría pleno sentido argumentar respecto a esta cuestión ${ }^{20}$.

Es importante señalar que si bien la razón de la no negociabilidad en ambos casos es la misma (la no disponibilidad), existen diferencias sustanciales entre ambos supuestos. Por ejemplo, supongamos que contamos con una máquina que nos permite producir lluvia. En este caso, puedo negociar la producción de ese fenómeno atmosférico. Pero lo que sigo sin poder negociar es el valor de verdad del enunciado. Creo que esto es así, por razones conceptuales, es decir, la noción de la verdad como correspondencia implica la no disponibilidad.

3. La tercera forma de interpretar la frase «uno no puede negociar sobre el clima» pondría el acento en el tema de las creencias; de esta manera diríamos que no es negociable mi creencia acerca de si está lloviendo.

Cabe resaltar la distinción que media entre la afirmación según la cual «la verdad de cierto enunciado no es negociable» de la afirmación acerca de la «no negociabilidad de las creencias en referencia a cierto hecho». Basta para ello admitir que el enunciado «cierto sujeto tiene razones para creer que es verdadero un hecho falso» tiene perfecto sentido. Por ejemplo, «Juan puede tener razones para creer que ayer llovió» y ser falso el enunciado «ayer llovió». Y esto con independencia de que la razón para sostener la no negociabilidad de las creencias vuelva a ser la misma; su no disponibilidad por parte del sujeto.

En un artículo titulado «Bargaining over Beliefs», GOODIN y BRENNAN ${ }^{21}$ han propuesto un modelo de negociación sobre creencias. La idea de estos autores es que el cambio de creencias está basado, generalmente, en la persuasión, es decir, en el proceso argumentativo. En estos contextos existen dos formas generales de modificar, condicionar o pulir las creencias de un individuo; mediante la aportación de nueva información disponible o mediante la técnica de ensayo-error. Sin embargo, a veces esto no es posible o los costos de este proceso son tan elevados que lo hacen irracional.

Un caso especial de este tipo de situaciones son los problemas en los que un conjunto de individuos tienen un fin común que sólo puede conseguirse si todos utilizan el mismo medio. Sin embargo, estos individuos discrepan acerca de cuál es el mejor medio para alcanzar dicho fin. Además, los costos de unificar dichas creencias (percepciones) son tan elevados que ponen en peligro la consecución del objetivo preferido por todos («convergencia interrumpida») 22 .

20 Por supuesto, aquí se presupone una determinada noción de verdad (verdad como correspondencia) pero no es claro que esto sea igual para otras nociones de verdad, por ejemplo, la verdad como convención. De todas maneras no me ocuparé aquí de las otras nociones de verdad.

${ }^{21}$ GoOdin y BrenNAn, 2001: «Bargaining over Beliefs», en Ethics, vol. 111, n. ${ }^{\circ} 2$, pp. 256 y ss.

22 Es importante no confundir lo que es un juego de cooperación (como el que presentan estos autores) con lo que es un juego de pura coordinación al que me he referido con anterioridad. La diferencia sustancial es que en los juegos de pura coordinación los sujetos son indiferentes respecto de las alternativas y respecto del modo de resolver la coordinación de éstas. Por el contrario, en los juegos cooperativos hay algún elemento en donde no es que haya indiferencia sino que hay un problema en referencia a la ordenación, en el caso de los autores, respecto de los medios de elección. En este sentido, un juego de pura coordinación excluye la noción de conflicto mientras que un juego cooperativo no. 
La idea que proponen estos autores es que en los casos de «convergencia interrumpida» la disputa sobre creencias puede ser resuelta mediante la negociación. En estos casos el acuerdo se produce, no en referencia a cuál es la creencia correcta, sino respecto a qué harán y por qué lo harán. El objeto del acuerdo en estos casos consiste, según GOODIN y BRENNAN, en tratar ciertas proposiciones «como si fuesen verdaderas» en referencia a una específica propuesta.

Sin embargo, creo que la propuesta de estos autores es incorrecta en la medida en que este planteamiento acerca de la negociabilidad de las creencias confunde, para decirlo rápidamente, la creencia con su aceptación, es decir, se confunde el enunciado «creo que ayer llovió» con el enunciado «aceptaré que ayer llovió». Si esto es así, creo que no cabe la negociación sobre las creencias. Ahora bien, lo que me parece interesante de la propuesta de estos autores es que ponen sobre la mesa una cuarta forma de analizar la afirmación de ELSTER.

4. Un cuarto, y último, ámbito de análisis de la negociabilidad es el tema de la aceptación. En lo que aquí interesa la característica más importante de la aceptación, frente a la creencia o a la verdad de un enunciado, es que ésta sí que es disponible, es decir, la aceptación es el resultado de una decisión. Siguiendo una ya clásica definición presentada por Jonathan COHEN, entenderé por aceptación «...de una proposición $p$ un estado mental consistente en tener o adoptar la política de usar $p$ en el razonamiento en algunos o en todos los contextos...» ${ }^{23}$.

Si admitimos esta definición, entonces negociar la aceptación de una proposición es negociar la política de usar dicha proposición en el razonamiento, esto es, en aquellas argumentaciones que se generan en el contexto de una negociación.

Podemos resumir lo hasta aquí dicho, pero ahora en referencia a las cuestiones de hecho de la siguiente forma:

1. No son susceptibles de negociación ni la verdad de un enunciado ni su creencia.

2. Una forma débil de entender la negociabilidad de una «cuestión de hecho» es vincularla con la negociabilidad de las acciones que pueden producir o condicionar ese hecho. Por ejemplo, siguiendo con ELSTER no sería negociable la tasa esperada de la inflación pero puedo negociar las acciones que condicionan o fijan dicha tasa.

3. Una segunda forma de entender la negociabilidad de una «cuestión de hecho» es relacionarla con la negociabilidad de la aceptación de una proposición. Volviendo sobre el mismo ejemplo, no es posible negociar la tasa pero sí es posible negociar la aceptación de la tasa esperada de inflación.

Me parece que es interesante resaltar que todos aquellos aspectos que quedan fuera de la negociación están vinculados con la «no disponibilidad» por parte del sujeto respecto a su conformación: ni los hechos, ni la verdad de los hechos, ni las creencias acerca de los hechos son elementos que puedan ser objeto de decisión por parte de un actor. Pero, si esto es así, entonces tampoco cabe sustentar la noción de conflicto, o al menos la noción que aquí he presentado en referencia a ellos. Esto concluye en mi opinión en la siguiente idea: no cabe predicar la negociación de estos elementos porque no hay posibilidad de dar cuenta de un conflicto de estos elementos. Puede haber discu-

23 L. J. Cohen, 1989: «Belief and Acceptance», en Mind, vol. XCVIII, n. ${ }^{\circ}$ 391, p. 368. 
siones, disputas, disparidad de opiniones u otros elementos controvertibles ${ }^{24}$ pero lo que no cabrá es la concurrencia de un conflicto.

Si esto es así, cabría entonces que aquellos que ponderan a la negociación negativamente porque excluyen estos ámbitos presenten una noción de conflicto y de solución de conflictos que permita considerar esta discusión.

Pero además, hay una segunda cuestión que me parece relevante vinculada con la introducción del elemento de la aceptación como un aspecto negociable. No es extraño que en diferentes ámbitos se sostenga que hay un prius lógico de los procesos deliberativos o de diálogo frente a los procesos negociales. Pero si se incorpora la idea de que es posible negociar la aceptación de una proposición en el razonamiento, entonces cabría pensar en diferentes conflictos (como el ejemplo de GOODIN y BRENAN) donde la negociación puede funcionar previamente a la utilización de otros métodos de solución de conflictos; negociamos qué es lo que vamos a aceptar en el discurso y una vez hecho esto podemos avanzar en el contexto de otras metodologías ${ }^{25}$.

\subsection{Los modos en la negociación}

Fuera del ámbito más especializado no es extraño hallar consideraciones que parecen reforzar la idea según la cual el único modo posible para un proceso de negociación es el compromiso. En este sentido, se defiende que todo proceso negociador, así como todos los objetivos de una negociación, concluyen o en la falta de una solución o en una solución que es necesaria y exclusivamente un resultado por compromiso, es decir, un resultado a partir de las concesiones recíprocas de los participantes.

Sin embargo, en mi opinión no es claro que la negociación excluya el resto de modos anteriormente señalados. Veamos esto con mayor detalle.

He indicado anteriormente que existen cuatro modos de finalización de un conflicto: la imposición, el desistimiento, la conversión y el compromiso. De estos cuatro modos considero que los tres últimos no resultan problemáticos en su relación con la negociación.

Compromiso: el compromiso es ciertamente uno de los modos tradicionales de resolución de conflictos. Sin embargo, hay que tener en cuenta que la trascendencia de este modo está muy vinculada con el tema de los modelos negociales al uso y en especial con el denominado modelo distributivo de negociación. Creo que algunas ideas generales servirán para entender mejor esta relación que algunos han considerado casi conceptual.

Las características primordiales de la propuesta distributiva de negociación son dos: por un lado, limita cualquier resolución a la definición del conflicto tal y como las partes lo presentan. Este límite se concreta fundamentalmente en dos elementos: a) los ob-

24 Puede verse una interesante discusión respecto de la diferencia entre estos conceptos en B. S. MAYER, 2004: Beyond Neutrality. Confronting the Crisis in Conflict Resolution, San Francisco: Jossey-Bass.

${ }_{25}$ Ya he defendido en otro lugar las ventajas que tiene esta estructura (negociación-diálogo) en referencia con un proceso de paz con una banda terrorista. Véase R. CALVO SOLER, 2006: La negociación con ETA. Entre la confusión y los prejuicios, Barcelona: Gedisa. 
jetivos de los actores, $\mathrm{y} b$ ) el objeto del conflicto. Y, por el otro lado, la propuesta de resolución sólo intentará fijar qué porcentaje de objeto o atributo corresponderá a cada uno de los actores según los objetivos por ellos expresados. El negociador no intenta redefinir la relación social entre los actores, ni establecer una ampliación de aquello que es objeto del conflicto a los efectos de procurar una plena satisfacción de los objetivos de cada actor.

Un ejemplo servirá para mostrar cómo funciona el elemento distributivo y el modo de compromiso en la negociación. Dos socios discuten por los beneficios generados en su empresa y por la cantidad que le corresponde a cada uno de ellos. Supóngase que los beneficios a repartir son de 5.000 euros. En este punto pueden pasar dos cosas: a) que cada actor defina su objetivo exclusivamente en términos de maximizar su beneficio en detrimento de la cantidad que le corresponde al otro actor («cuanto más se quede el otro menos me quedaré yo y viceversa»), o $b$ ) que cada actor defina su objetivo en términos de una distribución de la cantidad de objeto según algún criterio que ella defiende.

Este tipo de ejemplos paradigmáticos de la negociación potencia la idea de que el proceso negocial está vinculado con la solución por compromiso. Pero en la negociación también tienen su lugar otros modos.

Desistimiento: el desistimiento también puede formar parte de los modos de finalización del conflicto por el método negocial. Un ejemplo paradigmático sería la utilización de las promesas como un mecanismo para que el otro actor desista en la defensa de sus objetivos. Ahora bien, es importante recordar que, como indiqué anteriormente, el desistimiento no implica una modificación de los objetivos por parte del actor que desiste. En este caso uno de los negociadores cede en la defensa de éstos. Por ejemplo, supongamos un conflicto entre un padre y su hijo por la hora de llegada a casa. El hijo decide llegar a la hora que el padre le ha dicho porque le ha prometido que le comprará una moto si cumple con los horarios de llegada a casa. La muestra de que el objetivo del hijo de «llegar más tarde a casa» no ha cambiado es que si el padre retirase su promesa o si, simplemente, no la vinculase al tema del horario de llegada a casa, él llegaría más tarde. Este es el punto de diferencia con la conversión.

Conversión: si acepta como he sostenido anteriormente que la argumentación tiene o puede tener un lugar en el contexto de los procesos negociales, el ejemplo paradigmático de este modo de finalización sería el caso en que la propuesta (razones + oferta) convence al otro actor. El conflicto finaliza porque la modificación de los objetivos de uno de ellos supone la desaparición de la incompatibilidad entre ellos.

Ahora bien, quizás el tema más complejo a la hora de identificar los modos de finalización de un conflicto mediante un proceso de negociación es la cuestión de la imposición. En mi opinión, y en coherencia con la noción de negociación que he defendido en este trabajo, es posible vincular la noción de negociación a la de imposición. Creo que negar esto sería tanto como pensar que cuando uno negocia la compra de un piso debe estar dispuesto a comprometerse (a establecer concesiones) con todo lo que proponga el vendedor. Cuando se negocia cabe la posibilidad de imponer, y de hecho generalmente lo hacemos, ciertos elementos que han de ser aceptados por el otro actor. La inclusión en el concepto de negociación de los instrumentos de presión, como por ejemplo las amenazas, apunta precisamente a este aspecto posible de todo proceso ne- 
gociador. En este sentido, creo que no sería intuitivo negar la existencia de una negociación porque el vendedor impuso, mediante una amenaza, la condición de que el pago fuese en efectivo, o porque el padre amenazó con que a partir de cierta hora el hijo debería volver en taxi.

Lo único que, en mi opinión, requeriría una negociación es que todos los actores admitan la existencia de algunos puntos que, dentro de la mesa de negociación, no podrán ser resueltos mediante la imposición, el desistimiento o la conversión. Por ejemplo, no habría negociación si el vendedor presenta un contrato cerrado en el que no es posible modificar ninguno de los elementos que lo conforman o si el hijo desiste en todas y cada una de las condiciones del conflicto por la hora de llegada a casa. Tampoco creo que hubiese negociación, como señalé anteriormente, si todo lo que quiere hacer el vendedor es convencer a su cliente de lo bueno que es su producto y no considera posible la utilización de las amenazas, promesas u otros recursos. Pero todo esto es distinto a afirmar que negociar implica asumir que todas las cuestiones o aspectos del conflicto han de ser objeto de concesiones.

Podemos expresar estas ideas en los siguientes términos:

1. Es condición necesaria para hablar de negociación el modo del compromiso (concesión).

2. No es condición suficiente para negar la existencia de un proceso de negociación que haya imposición, desistimiento o conversión.

En este sentido, podemos negociar mientras que imponemos, desistimos o nos convertimos durante el proceso. Por supuesto que, en el juego negociador, también es posible oponerse a las reclamaciones sustentadas en estos tres modos de finalización del conflicto. Pero eso es parte del proceso de negociación.

Creo que la razón de que esto funcione es que la negociación opera sobre las voluntades de los actores como único sustento de la solución del conflicto. Y, por esta razón, la inclusión de la imposición, del desistimiento o de la conversión, junto con la ineludible concesión, no impide el proceso negociador. 